\title{
Títulos de anticuerpos contra Leptospira sp., en primates del zoológico Matecaña, Pereira, Colombia
}

\section{Antibody titers against Leptospira sp., in primates from Matecaña zoo, Pereira, Colombia}

\author{
Marlyn Romero P, ${ }^{1 *}$ M.Sc, Miryam Astudillo $\mathrm{H}^{2}{ }^{2}$ M.Sc, Jorge Sánchez ${ }^{1}{ }^{1}$ M.Sc, Lina \\ González G, ${ }^{1}$ MVZ, Néstor Varela A, ${ }^{3}$ Esp.
}

\begin{abstract}
1Universidad de Caldas, Facultad de Ciencias Agropecuarias, Departamento de Salud Animal, Grupo de Investigación CIENVET. Manizales, Colombia. ²Universidad del Valle, Departamento de Microbiología, Cali, Colombia. ${ }^{3 Z}$ Zoológico Matecaña, Pereira, Colombia. *Correspondencia: marlyn. romero@ucaldas.edu.co
\end{abstract}

Recibido: Febrero de 2011; Aceptado: Diciembre de 2011.

\section{RESUMEN}

Objetivo. Identificar los serovares más frecuentes de Leptospira sp., por la técnica de Microaglutinación (MAT) en una población de primates neotropicales, mantenida en condiciones de cautiverio, y evaluar por microscopía de campo oscuro la presencia de espiroquetas en aguas para el consumo de los animales. Materiales y métodos. Se realizó un estudio de prevalencia de punto en 65 monos neotropicales, mediante la técnica de Microaglutinación Macroscópica (MAT) usando un cepario de referencia conformado por 21 serovares de Leptospira sp., y la observación de espiroquetas en el agua mediante microscopía de campo oscuro. Resultados. La seroprevalencia de Leptospira sp., en los monos neotropicales fue del $41.5 \%$ (27/65). Los serovares de Leptospira Autumnalis (25\%), Ranarum $(22.9 \%)$, Icterohaemorrhagiae (12.5\%), Pomona (12.5\%) y Australis $(10.4 \%)$ fueron los más aglutinados por los sueros. Los monos ardilla (Saimiri sciureus), lanudo (Lagothrix lagotricha) y tití pielroja (Saguinus oedipus) fueron seronegativos. Se observaron espiroquetas en el agua evaluada. Conclusiones. Se evidencia la circulación de Leptospira sp., en el zoológico, siendo necesario la implementación de estrategias de vigilancia epidemiológica activa y programas de promoción.

Palabras clave: Monos, seroprevalencia, zoológico (Fuente: $C A B$ ). 


\section{ABSTRACT}

Objectives. To identify the most frequent serovars of Leptospira sp., in a captive neotropical primate population using the Microagglutination test (MAT), and to evaluate the presence of spirochetes in the drinking water of enclosures using dark-field microscopy. Materials and methods. A point prevalence study was performed in 65 neotropical monkeys using the microagglutination test (MAT) and 21 Leptospira sp., reference strains; observation of spirochetes in water was performed using the dark-field microscopy. Results. The Leptospira sp., seroprevalence was $41.5 \%$ (27/65) in neotropical monkeys. Leptospira serovars showing important agglutination were Autumnalis (25\%), Ranarum (22.9\%), Icterohaemorrhagiae (12.5\%), Pomona (12.5\%) and Australis $(10.4 \%)$. Squirrel monkey (Saimiri sciureus), woolly monkey (Lagothrix lagotricha) and cotton-top tamarin (Saguinus oedipus) had non-reactive sera. Spirochetes in drinking water of enclosures were observed. Conclusions. This study shows evidence of the circulation of Leptospira sp., inside the zoo setting, revealing the need of implementing active epidemiological surveillance strategies and educational programs.

Key words: Monkeys, seroprevalence, zoo (Source: $C A B$ ).

\section{INTRODUCCIÓN}

La Leptospirosis es una enfermedad zoonótica bacteriana de importancia y distribución global (1). Presenta un comportamiento endémico, pero se reportan brotes epidémicos en varios continentes; siendo considerada una enfermedad reemergente en la actualidad $(1,2)$. Las enfermedades infecciosas emergentes y reemergentes tienen dos implicaciones biológicas de importancia; en primer lugar, muchas especies de vida silvestre son reservorios de patógenos que amenazan la salud de las personas y de los animales domésticos; y segundo, constituyen una amenaza sustancial para la conservación de la biodiversidad global (3). Por lo tanto, su investigación puede beneficiar los esfuerzos en la conservación de la vida silvestre y proveer una conexión entre los estudios serológicos y la necesidad de la detección, identificación y vigilancia epidemiológica oportuna de estas enfermedades $(4,5)$.

En la cadena epidemiológica de la leptospirosis participan hospederos de mantenimiento y hospederos definitivos (5). Los primeros se definen como las especies animales en las cuales la infección es endémica, usualmente transmitida de individuo a individuo por contacto directo. A diferencia, los hospederos accidentales (como el hombre) se infectan por contacto indirecto por contaminación ambiental con orina de animales infectados (1). Esta última vía se presenta a través de la ingestión de agua o alimento contaminado, y por contacto con mucosas o la piel (2).

Es poco frecuente el reporte de leptospirosis en primates no humanos y por lo tanto, la presentación de la enfermedad ha sido poco estudiada (6), a pesar de que se ha comprobado que actúan como portadores renales del patógeno, lo cual los convierte en un riesgo para los animales de zoológicos y de laboratorio, al igual que para los trabajadores, los veterinarios y los demás primates $(7,8)$. Los síntomas de leptospirosis observados en infecciones naturales de primates generalmente son leves, pasan desapercibidos; limitándose a respuestas febriles, conjuntivitis e inquietud (9). Por otra parte, la respuesta humoral es detectada sólo en períodos cortos de tiempo (7). Sin embargo, en condiciones de cautiverio, el frágil equilibrio entre los agentes infecciosos y los hospederos se rompe, debido a problemas como la desnutrición y el estrés crónico (fruto de ambientes inadecuados, totalmente distintos a los ambientes naturales), el desconocimiento de las necesidades básicas de las diferentes especies, la limitación espacial que los puede predisponer a una reinfección, así como, las alteraciones orgánicas y del comportamiento, que favorecen el establecimiento de enfermedades y el desarrollo de cuadros clínicos más severos $(4,6,9)$.

A pesar de estas limitaciones, los zoológicos ofrecen oportunidades para el estudio de la leptospirosis en situaciones controladas y son fuente importante de información para la investigación epidemiológica de enfermedades transmisibles (8). Teniendo en cuenta la escasa información sobre la epidemiología de la leptospirosis en poblaciones silvestres en zoológicos colombianos, el presente trabajo tuvo como objetivo realizar una evaluación serológica para establecer los serovares más frecuentes de Leptospira sp., por la técnica de microaglutinación (MAT), en una población de primates neotropicales mantenida en condiciones 
de cautiverio y evaluar por microscopía de campo oscuro la presencia de espiroquetas en aguas de bebida de los animales.

\section{MATERIALES Y MÉTODOS}

Localización. El zoológico de Matecaña se encuentra localizado en la ciudad de Pereira, departamento de Risaralda, Colombia. El municipio se encuentra a $4^{\circ} 49$ minutos de latitud norte y $75^{\circ} 45$ minutos de latitud oeste de Greenwich, con una temperatura promedio de $21^{\circ} \mathrm{C}$.

Primates no-humanos. La población de primates neotropicales evaluados está conformada por 65 individuos, pertenecientes a nueve especies taxonómicas: Ateles fusciceps, Cebus albifrons, Cebus apella, Cebus capucinus, Saimiri sciureus, Ateles hybridus, Lagothrix lagotricha, Saguinus oedipus y Saguinus leucopus.

Normas de ética. El estudio fue aprobado por el Comité de Ética de la Universidad de Caldas y contó con el consentimiento informado para la toma de las muestras.

Muestras sanguíneas de los primates. Los monos neotropicales fueron sometidos a un procedimiento de contención química, utilizando como protocolo anestésico una mezcla de Ketamina $(10 \mathrm{mg} / \mathrm{kg})$ y Xilacina $(1 \mathrm{mg} / \mathrm{kg})$ vía intramuscular. Se siguió el pulso, la frecuencia respiratoria y cardíaca, la relajación muscular, analgesia, reflejos (palpebral, corneal, interdigital y genital) cada 10 minutos. La vena de elección para la colecta, cantidad de sangre y el tamaño de la aguja, se seleccionó de acuerdo con la edad y la masa corporal de cada animal. Las muestras de sangre fueron tomadas con tubo Vacutainer sin anticoagulante, luego de un reposo de 10 minutos se centrifugaron a $3000 \mathrm{rpm}$ durante cinco minutos y el suero obtenido se almacenó a $-20^{\circ} \mathrm{C}$ hasta su procesamiento en el laboratorio.
Muestreo y análisis del agua. Se tomaron 10 $\mathrm{ml}$ de agua de los bebederos de cinco locaciones de monos neotropicales y una muestra del tanque distribuidor del zoológico Matecaña, las cuales fueron analizadas mediante microscopía de campo oscuro.

Técnica de Microaglutinación MAT. EI procesamiento de las muestras se efectuó en el laboratorio de Diagnóstico de Leptospirosis de la Universidad del Valle (Cali); laboratorio que participa en el control externo de la MAT realizado por la Sociedad Internacional de Leptospirosis. El mantenimiento de las cepas y el manejo de la técnica se realizó bajo los parámetros convencionales (10), utilizando un cepario de referencia suministrado por el Laboratorio Internacional de Referencia para el Diagnóstico de la Leptospirosis del Royal Tropical Institute, Amsterdam (Holanda).

Los 21 serovares de Leptospira evaluados fueron: Bataviae, Mini, Autumnalis, Sutumnalis, Canicola, Shermani, Icterohaemorrhagiae, Cynopteri, Australis, Celledoni, Gryppotyphosa, Hebdomanis, Javanica, Manhao, Pomona, Pyrogenes, Sejroe, Tarassovi, Bataviae, Patoc, Hurstbridge y Djasiman. Se consideraron como positivos los sueros con títulos $\geq 1: 50$. Se determinó el título de anticuerpos utilizando microscopio de campo oscuro, objetivo $25 \mathrm{X}$, cuando al comparar con un control de antígeno, el 50\% o una proporción mayor de las leptospiras estaban aglutinadas en una dilución del suero entre $1: 50$ y $1: 3200$.

Análisis estadístico. Se utilizó el paquete estadístico Epidemiology Program Office Epi Info ${ }^{T M}$ versión 6.

\section{RESULTADOS}

La seroprevalencia de Lectospira sp., en la población de primates neotropicales evaluados fue del $41.5 \%$ (27/65). Los monos neotropicales mono ardilla (Saimiri sciureus), mono lanudo (Lagothrix lagotricha) y tití pielroja (Saguinus oedipus) fueron seronegativos (Tabla 1 ).

Tabla 1. Especies de primates neotropicales evaluados y serovares seroreactivos en el Zoológico Matecaña, Pereira, Colombia.

\begin{tabular}{lccl}
\multicolumn{1}{c}{ Primates } & Pos & Exa & \\
\hline Ateles fusciceps (Mono araña negro) & 6 & 19 & Autumnalis, Canicola, Celledoni, Icterohaemorrhagiae, Lau, Patoc Pomona. \\
Ateles hybridus (Mono cornudo) & 2 & 6 & Autumnalis, Icterohaemorrhagiae \\
Cebus apella (Mono capuchino) & 7 & 14 & Australis, Autumnalis, Ranarum, Sarmin \\
Cebus capucinus (Mono cariblanco) & 1 & 3 & Australis, Autumnalis, Ranarum \\
Cebus albifrons (Mono ardilla) & 6 & 6 & Australis, Autumnalis, Cynopteri, Icterohaemorrhagiae, Pomona, Ranarum \\
Saimiri sciureus (Mono araña mulato) & 0 & 4 & \\
Lagothrix lagotricha (Mono lanudo) & 0 & 2 & \\
Saguinus oedipus (Tití piel roja) & 0 & 6 & \\
Saguinus leucopus (Tití gris) & 5 & 5 & Cynopteri, Ranarum \\
\hline Total & $\mathbf{2 7}$ & $\mathbf{6 5}$ & \\
\hline Pos Positivos; Exa & & &
\end{tabular}

Pos = Positivos; Exa = Examinados 
Tabla 2. Frecuencia de aglutinación anti-Leptospira sp. en sueros de primates en cautiverio del Zoológico Matecaña, Colombia, 2010.

\begin{tabular}{|c|c|c|c|c|c|c|c|c|}
\hline \multirow[t]{2}{*}{ Serovar } & \multicolumn{7}{|c|}{ TÍTULO } & \multirow[t]{2}{*}{$\%$} \\
\hline & 50 & 100 & 200 & 400 & 800 & $>1600$ & Total & \\
\hline Australis & 04 & 00 & 01 & 00 & 00 & 00 & 05 & 10.4 \\
\hline Autumnalis & 07 & 04 & 00 & 01 & 00 & 00 & 12 & 25 \\
\hline Bataviae & 00 & 00 & 00 & 00 & 00 & 00 & 00 & 0 \\
\hline Canicola & 00 & 00 & 01 & 00 & 00 & 00 & 01 & 2.1 \\
\hline Celledoni & 00 & 00 & 00 & 00 & 00 & 00 & 00 & 0 \\
\hline Cynopteri & 02 & 00 & 02 & 00 & 00 & 00 & 04 & 8.3 \\
\hline Djasiman & 00 & 00 & 00 & 00 & 00 & 00 & 00 & 0 \\
\hline Gryppotyphosa & 00 & 00 & 00 & 00 & 00 & 00 & 00 & 0 \\
\hline Hebdomanis & 00 & 00 & 00 & 00 & 00 & 00 & 00 & 0 \\
\hline Hursbridge & 00 & 00 & 00 & 00 & 00 & 00 & 00 & 0 \\
\hline Icterohaemorrhagiae & 01 & 00 & 02 & 01 & 01 & 01 & 06 & 12.5 \\
\hline Lau & 00 & 00 & 01 & 00 & 00 & 00 & 01 & 2.1 \\
\hline Mini & 00 & 00 & 00 & 00 & 00 & 00 & 00 & 0 \\
\hline Manhao & 00 & 00 & 00 & 00 & 00 & 00 & 00 & 0 \\
\hline Panama & 00 & 00 & 00 & 00 & 00 & 00 & 00 & 0 \\
\hline Pomona & 01 & 02 & 00 & 01 & 01 & 01 & 06 & 12.5 \\
\hline Ranarum & 05 & 06 & 00 & 00 & 00 & 00 & 11 & 22.9 \\
\hline Sarmin & 01 & 00 & 00 & 00 & 00 & 00 & 01 & 2.1 \\
\hline Serjoe & 00 & 00 & 00 & 00 & 00 & 00 & 00 & 0 \\
\hline Shermani & 00 & 00 & 00 & 00 & 00 & 00 & 00 & 0 \\
\hline Patoc & 01 & 00 & 00 & 00 & 00 & 00 & 01 & 2.1 \\
\hline Total & 22 & 12 & 07 & 03 & 02 & 02 & 48 & 100 \\
\hline$\%$ & 45.8 & 25 & 14.6 & 6.2 & 4.2 & 4.2 & & \\
\hline
\end{tabular}

Los serovares de Leptospira Autumnalis (25\%), Ranarum (22.9\%), Icterohaemorrhagiae $(12.5 \%)$, Pomona (12.5\%) y Australis (10.4\%) fueron los más aglutinados por los sueros de los primates neotropicales (Tabla 2 ).

\section{DISCUSIÓN}

No fue posible determinar las vías de transmisión y las fuentes de infección relacionadas con la presencia de animales positivos para Leptospira sp., en el zoológico Matecaña. Sin embargo, de las seis muestras de agua evaluadas por microscopía de campo oscuro, en tres de éstas se visualizaron formas microscópicas compatibles con Leptospira sp., pero por la baja especificidad de la técnica, no fue posible establecer con certeza su naturaleza patógena o saprófita (2). Este hallazgo sugiere que es necesario realizar evaluaciones complementarias para dilucidar su clasificación, teniendo en cuenta que las leptospiras patógenas pueden sobrevivir en agua por muchos meses, dependiendo de la temperatura, el $\mathrm{pH}$, la salinidad o el grado de contaminación (11). Asimismo, trabajos realizados en aguas de lagos del zoológico de la Plata (Argentina) demostraron la presencia de leptospiras, haciendo evidente el papel del agua en la epidemiología de la enfermedad (12).

Es importante resaltar que en el año 2007 en el zoológico Matecaña se presentó un brote de leptospirosis en animales y en el personal; por lo tanto, la alta seroprevalencia de punto de leptospirosis encontrada (41.5\%) en este estudio indica que los primates evaluados estuvieron expuestos a la infección por Leptospira sp., y que existe un riesgo de transmisión a las especies susceptibles, incluidos los operarios responsables de su cuidado; teniendo en cuenta que se ha sugerido su papel como portadores renales del patógeno (13).

La seroprevalencia fue superior a la reportada en los zoológicos de Rio de Janeiro y de Aracaju $(6,8)$, así como a la encontrada en 286 muestras de Cebus apella $(16.1 \%)$ capturados en un ambiente natural del estado de Tocantins, Brasil (14); e inferior a la detectada en el zoológico de Sao Paulo del 65\% $(13 / 21)(15)$. Estas diferencias se pueden deber a factores relacionados con el agente, los hospederos, el ambiente, el manejo y a características propias de la técnica MAT.

Los títulos de los sueros evaluados para los distintos serovares de Leptospira interrogans, variaron entre $1: 50$ y $1: 1600$ (Tabla 2). Predominaron los títulos bajos de 1:50 (45.8\%), lo que sugiere, que sólo hubo o hay infección y el título de inmunoglobulinas en el momento del examen corresponde a un punto de la curva de anticuerpos. Asimismo, los animales portadores de un serovar presentaron títulos bajos. La MAT detecta especialmente inmunoglobulina IgM, lo cual indica infección inicial y por consiguiente no siempre están correlacionados con los signos 
clínicos, los cuales muchas veces se presentan cuando los niveles de IgM han descendido.

Los títulos serológicos $\geq 100$ (54.2\%) evidencian que los animales padecieron la enfermedad o que la infección está en curso. Los títulos $\geq 1600$ (4.2\%), pueden indicar una infección activa, sin presentar manifestaciones clínicas, siendo viable también la eliminación del agente por la orina (9). En este caso es necesario realizar estudios complementarios con el fin de confirmar el diagnóstico mediante técnicas como el aislamiento, tipificación y análisis molecular e implementar el tratamiento respectivo.

Las investigaciones serológicas sobre la leptospirosis en primates neotropicales en cautiverio han presentado resultados variables. Los serovares de Leptospira sp., más frecuentemente encontrados en primates de Brasil fueron Castellonis, Copenhageni, Grippotyphosa (15), resultados diferentes a los hallados en el presente estudio, en donde predominaron Autumnalis, Ranarum, Icterohaemorrhagiae, Pomona y Australis, que evidencian la presencia de leptospiras patógenas en zoológicos.

Se ha descrito un amplio rango de reservorios de los serovares Autumnalis y Australis como roedores silvestres, prociónidos (mapache), mustélidos (comadreja y zorrillo), roedores sinantrópicos (Rattus norvegicus, Rattus rattus, Mus musculus), porcinos y bovinos (16). En este caso, las condiciones de cautiverio que favorecen el contacto estrecho entre animales con orígenes ecológicos y antecedentes epidemiológicos disímiles, pueden contribuir a la exposición de los animales con diferentes serovares (17).

El serovar Icterohaemorrhagiae ha sido frecuentemente identificado en estudios serológicos en casos confirmados y probables de leptospirosis en seres humanos en Colombia $(18,20)$. Asimismo, la infección por éste serovar ha sido comprobada por serología y genotipificación en un brote de leptospirosis severa en monos capuchinos confiscados y mantenidos en un centro de rehabilitación de fauna silvestre colombiano (21). El serovar Icterohaemorrhagiae fue aislado de 3 casos fatales de leptospirosis presente en macacos de Gibraltar en cautiverio (Macaca sylvanus) en el parque zoológico Nacional de Washington, D.C. (13), siendo descrito además en varias especies de mamíferos en el zoológico de Rio de Janeiro (17).

Estos hallazgos permiten sugerir que a pesar de que los primates evaluados en el presente trabajo tienen hábitos arborícolas en su medio natural, se ven forzados a tener mayor contacto con el piso de las instalaciones, debido a las condiciones de cautiverio; aumentando el riesgo de contacto con roedores, o con ambientes y alimentos contaminados con su orina (21), convirtiéndose en una fuente de infección de este serovar, teniendo en cuenta que los roedores, especialmente los sinantrópicos como la rata parda (Rattus norvergicus), son reservorios importantes (8).

Como medidas de control se pueden implementar: la utilización de barreras físicas que eviten el ingreso de los roedores, mejorar el manejo de los restos de alimentos en los recintos, suministrar el agua en recipientes localizados en estructuras elevadas, proporcionar suficientes plataformas para que los animales descansen, limpiar frecuentemente el piso de las instalaciones, recubrimiento del piso con cemento para evitar acumulaciones de agua, y mantenimiento preventivo de instalaciones, entre otras medidas (21).

La alta seroprevalencia de Leptospira sp., en el presente estudio sugiere que se deben incluir los primates no humanos en los programas de vigilancia epidemiológica activa de la infección en poblaciones silvestres mantenidas en condiciones naturales y en cautiverio; debido a que los médicos veterinarios por lo general asumen que los monos no son susceptibles a la infección por Leptospira; apreciación que puede conducir a diagnósticos y tratamientos errados .

Adicionalmente, la infraestructura de los zoológicos puede ser propicia para la sobrevivencia de Leptospira sp., en el ambiente, como consecuencia de la presencia de áreas adyacentes muy arborizadas, que en ocasiones entorpecen la entrada de la radiación solar a los recintos de los animales, o mantienen una humedad elevada; especialmente en los períodos de verano y de alta pluviosidad, siendo común la formación de charcos de agua en las instalaciones, aspecto que es importante controlar (22).

Finalmente se evidenció la circulación de Leptospira sp., en los primates neotropicales del zoológico Matecaña; siendo necesario fortalecer los programas de promoción y prevención de la leptospirosis para evitar la transmisión de la infección a otras especies animales y a los funcionarios.

\section{Agradecimientos}

A la Vicerrectoría de Investigaciones y Postgrados de la Universidad de Caldas, al Zoológico Matecaña de Pereira y al Laboratorio de Diagnóstico de Leptospirosis de la Universidad del Valle, por la financiación de esta investigación. 


\section{REFERENCIAS}

1. Bharti A, Nally J, Ricaldi JN, Matthias MA, Diaz $M$, Lovett MA et al. Leptospirosis: a zoonotic disease of global importance. Lancet Infect Dis 2003; 3(12):757-771.

2. Levett PN. Leptospirosis. Clin Microbiol Rev 2001; 14(2):296-326.

3. Daszak $P$, Cunningham $A$, Hyatt $A$. Anthropogenic environmental change and the emergence of infectious diseases in wildlife. Acta Trop 2001; 78(2):103- 116.

4. Cutler SJ, Fooks AR, van der Poel WHM. Public Health threat of new, reemerging and neglected zoonoses in the industrialized world. Emerg Infect Dis 2010; 16(1):1-7.

5. Sleeman J. Wildlife zoonoses for the veterinary practitioner. J Exotic Pet Med 2006; 15(1):25-32.

6. Lilenbaum W, Monteiro RV, Albuquerque CE, Ristow P, Fraguas S, Cardoso VSet al. Leptospiral antibodies in wild felines from Rio de Janeiro Zoo, Brazil. Vet J 2004; 168(2):191-193.

7. Scarcelli E, Piatti RM, Fedullo JDL, Faiçal S, Cardoso MV, Castro V et al. Leptospira spp. detection by polymerase chain reaction (PCR) in clinical samples of captive blackcapped capuchin monkey (Cebus apella). Braz J Microbiol 2003; 34(2):143-146.

8. Pimentel JS, Gennari SM, Dubey J, Marvulo MFV, Vasconcellos SA, Morais ZM et al. Inquérito sorológico para toxoplasmose e leptospirose em mamíferos selvagens neotropicais do zoológico de Aracaju, Sergipe. Pesq Vet Bras 2009; 29(12):1009-1014.

9. Silva C, Gírio RJ da S, Guerra-Neto G, Brich M, Santana LA, Amâncio FH et al. Anticorpos anti-Leptospira spp. em animais selvagens do zoológico municipal de Ribeirão Preto, Estado de São Paulo State, Brazil. Braz J Vet Res Anim Sci 2010; 47(3):237-242.

10. Astudillo M, González A, Batista N, Mirabal M, Menéndez J. Estudio seroepidemiológico de la leptospirosis humana en el departamento del Valle del Cauca, Colombia. Rev Cubana Med Trop 2009; 61(2):1-10.
11. Brod CS, Aleixo JA, Jouglard SDD, Fernandes $\mathrm{CPH}$, Teixeira JLR, Dellagostin OA. Evidência do cão como reservatório da leptospirose humana: isolamento de um sorovar, caracterização molecular e utilização em inquérito sorológico. Rev Soc Bras Med Trop 2005; 38(4):294-300.

12. Gatti M, Arias D, Rosetti C, Selva S, Copes J, Laplace $R$ et al. Investigación de Leptospiras en lagos del zoológico de la Plata, Argentina. Analecta Vet $2004 ; 24(1): 18-20$.

13. Morosini de Andrade T. Títulos de anticorpos contra Leptospira spp e análise bioquímica no soro sangüíneo em macaco Prego (Cebus apella nigritus). [Tesis de Doctorado]. Jaboticabal, Brasil: Universidad Estatal Paulista "Júlio de Mesquita Filho", Faculdade de Ciências Agrárias e Veterinárias; Area Medicina Veterinária Preventiva; 2007.

14. Souza Júnior MF, Lobato ZIP, Lobato FCF, Moreira EC, de Oliveira RR, Leite GG et al. Presença de anticorpos da clase IgM de Leptospira interrogans em animais silvestres do Estado do Tocantins, 2002. Rev Soc Bras Med Trop 2006; 39(3):292-294.

15. Corrêa SHR, Vasconcellos SA, Morais Z, Teixeira AA, Dias RA, Guimarães MADB et al. Epidemiologia da leptospirose em animais silvestres na Fundacão Parque Zoológico de São Paulo. Braz J Vet Res Anim Sci 2004; 41(3):189-193.

16. Ward MP. Clustering of reported cases of leptospirosis among dogs in the United States and Canada. Prevent Vet Med 2002; 56:215-226.

17. Lilenbaum W, Monteiro RV, Ristow P, Fraguas $\mathrm{S}$, Cardoso VS, Fedullo LPL. Leptospirosis antibodies in mammals from Rio de Janeiro Zoo, Brazil. Res Vet Sci 2002; 73(12):319-321.

18. Romero MH, Sánchez JA, Hayek LC. Leptospirosis, brucelosis y toxoplasmosis: zoonosis de importancia en población ocupacionalmente expuesta. Biosalud 2008; 7(1):21-27. 
19. Romero MH, Sánchez JA, Hayek LC. Prevalencia de anticuerpos contra Leptospira en población urbana humana y canina del departamento del Tolima. Revista de Salud Pública 2010; 12(2):268-275.

20. Agudelo-Flórez $P$, Restrepo-Jaramillo BN, Arboleda-Naranjo M. Situación de la leptospirosis en el Urabá antioqueño colombiano: estudio seroepidemiológico y factores de riesgo en población general urbana. Cad Saúde Pública 2007; 23(9):2094-2102.
21. Szonyi B, Agudelo-Flórez $P$, Ramírez $M$, Moreno N, Ko Albert I. An outbreak of severe leptospirosis in capuchin (Cebus) monkeys. Vet J $2011 ; 188(2): 237-9$.

22. Neto-Guerra G. Freqüência de anticorpos contra Leptospira spp. em felídeos neotropicais em cativeiro no Brasil. [Tesis Mestre]. Jaboticabal, Brazil: Universidad Estatal Paulista "Júlio de Mesquita Filho", Faculdade de Ciências Agrárias e Veterinárias, Departamento Patología Animal; 2006. 